\title{
(Probably) Not Companions in Guilt
}

\author{
Sharon Berry
}

\begin{abstract}
In this paper, I will attempt to develop and defend a common form of intuitive resistance to the companions in guilt argument. I will argue that (contra the companions in guilt argument) one can reasonably believe there are promising solutions to the access problem for mathematical realism that don't translate to moral realism. In particular, I will suggest that the structuralist project of accounting for mathematical knowledge in terms of some form of logical knowledge offers significant hope of success while no analogous approach offers such hope for moral realism ${ }^{1}$.
\end{abstract}

\section{Introduction}

If moral realism is correct, what can explain human accuracy about moral facts? The supposed match between moral facts and our beliefs about them can seem quite mysterious. This worry is sometimes called the 'access problem' (for moral realism) and is a popular motivation for moral anti-realism.

One popular response to this challenge is the Companions in Guilt argument, which maintains that accepting mathematical realism generates an exactly parallel access problem. If this analogy holds up, then one cannot reject moral realism (purely) on the basis of the access problem above, while accept-

\footnotetext{
${ }^{1}$ Thanks to Ned Hall, Warren Goldfarb, Peter Koellner, Bernard Nickel, David Enoch, Stuart Shapiro, Tom Donaldson, Rachael Briggs, Selim Berker, Daniel Nolan, Kim Sterelny,Silvia Jonas, the audiences of NYU and Cambridge graduate conferences and (as always) Peter Gerdes for helpful comments on this project.
} 
ing mathematical realism. But, many philosophers who happily take anti-realist positions about morals are loath to do so about mathematics.

I will suggest that a justification for this differential treatment can be found in the following difference between mathematical and moral investigation. Mathematicians (and most philosophers of a realist persuasion) are willing to accept that investigations of alternate logically coherent mathematical structures would yield knowledge of mathematical truths of equal metaphysical status. For example, mathematicians could decide to investigate the natural numbers under plus and times except for 17 (though such a structure is unlikely to be interesting $)^{2}$. In contrast, moral realists hold that almost no alternate practice of deciding what behaviors count as "permissible" would yield true beliefs about some other concept of equal metaphysical status (and the same holds for other moral vocabulary). Baring the success of a very controversial Kantian program, this implies that very few logically coherent moral practices would yield truths of equal status. Thus, there seems to be a close relationship between mathematics and logic (specifically logical coherence) which makes reducing access worries about mathematics to access worries about logical coherence a promising project, in a way that the analogous reduction of moral facts to logical coherence facts it is not.

The strategy of trying to explain mathematics via 'logic' and thereby address access worries, goes back to Frege[16] and Hilbert[44], but aggressive claims in this area have left something of a philosophical stain on this approach. However, I will suggest that an appropriately modest (and modern) version of this strategy actually fares quite well and need not be in tension with metamathematical results that posed problems for previous proposals (e.g., Gödelian proof transcendence), and the philosophical insights derived from them. In partic-

\footnotetext{
${ }^{2}$ Of course, we must give some other behavior for + and $*$ in those cases where those operations would have yielded 17. Perhaps we might define $x+y$ and $x * y$ to equal 13 and 22 respectively if they would have yielded 17 in the natural numbers.
} 
ular, we will see that a range of popular contemporary philosophies of mathematics (within what I will call the structuralist consensus) reconcile realism about mathematics (including proof transcendent mathematical facts and even, in many cases, the literal existence of mathematical objects) with the idea that almost any coherent mathematical structure can be posited.

I will then delineate a strategy for solving the residual access problem for knowledge of logical coherence that I believe to be quite promising. In so doing, I hope to show that, plausibly, the mathematical realist can explain access to mathematical facts through access to facts about logical coherence, while the moral realist cannot successfully employ an analogous strategy.

Admittedly, this point alone does not suffice to show that the access problem for moral realism is (ultimately) worse than that for mathematical realism. For maybe there is some other, even better, strategy for addressing access worries about morals (unrelated to exploiting a connection to logic) which has no ana$\log$ for mathematics. Or maybe current appearances regarding the prospects for Kantianism about morals and/or the 'structuralist consensus' regarding mathematics are deceptive. But it does suffice to explain and defend the current position of many philosophers, who accept mathematical realism while rejecting moral realism on the basis of access worries. Contrary to the Companions in Guilt argument no hypocrisy is needed to take this position, for there are credible lines of attack on the mathematical access problem which appear (at least in light of the current development of relevant philosophical research programs) to be much less promising when applied in the moral domain ${ }^{3}$.

\footnotetext{
${ }^{3}$ In [7], Justin Clarke-Doane briefly advocates a related idea: that some kind of deflationary answer to access worries (exploiting the idea that different mathematical posts would have succeeded) is more plausible in the mathematical case than the moral case. However, it is important to note the kind of mathematical deflationism Clarke-Doane considers is more radical than the modal structuralist consensus I consider (and anything I view as plausible). Clarke-Doane only suggests that anti-realists about mathematics can use the strategy he outlines to help with access worries. In particular, he takes the strategy he suggests to involve (somehow) denying the "objectivity" of mathematics. In contrast, I present reasons for thinking that mathematics faces less of an access problem which are compatible with
} 


\section{Setting up the Question}

Let me begin by clarifying how I will understand the Companions in Guilt thesis and what it means for a theory to face an access problem.

Mackie introduced the idea of a 'Companions in Guilt' argument in [32] pg 39, as a possible response to his argument from queerness, which contends that moral realist facts would have to be somehow deeply (and implausibly) metaphysically or epistemologically different from all other kinds of facts which we reasonably accept. In this paper, I will be considering the more recent formulation of the Companions in Guilt argument given in [6], which appeals to a comparison with mathematics to diffuse access worries about moral realism. So let me now clarify what I mean by 'an access problem', and what it means for one theory's access problem to be worse than another's.

In Realism, Mathematics and Modality[13] Hartry Field influentially proposes that we should think of the access problem for (mathematical) realists as arising from a challenge for the realist to "explain how our beliefs about [mathematical objects] can so well reflect the facts about them" in some internally coherent fashion. More specifically, Field demands that we explain the truth of 'reliably, if mathematicians believe that $\phi$ then $\phi$ ', for various mathematical statements $\phi$. And he notes that, "[I]f it appears in principle impossible to explain this, then that tends to undermine ... belief in mathematical entities, despite whatever reason we might have for believing in them." It has been very popular to construe access worries about morals, metaphysical possibility, mathematical truth-value realism, aesthetics etc. analogously. On this view, access worries provide us ceterus paribus reason for rejecting a theory (in this

accepting realism and objectivity about mathematics.

Clarke-Doane also seems to think that our current total conception of the sets (not just the first order fragment of it embodied in the ZFC axioms) can be coherently extended by adding $\mathrm{AC}$ or $\neg \mathrm{AC}$ - something which I reject, and which he gives no argument for.

Finally, Clarke-Doane says nothing about how we are able to reliably avoid making incoherent mathematical posits which I take to be a key part of addressing access worries. 
case realism about mathematical objects), by appealing to a kind of cumulative gestalt impression that no adequate answer to this explanatory demand seems possible.

Accordingly, I will say that the access worry for realism about a given domain is worse, to the extent that there seems to be more reason to think a satisfying explanation of human accuracy about that domain is impossible. And we can think of Companions in Guilt arguments as saying that the Moral Realists' access problem is no worse than the Mathematical Realists' access problem because any roadblocks to explaining access to morals also apply to the mathematical case.

In this paper I will attack the above companions in guilt argument by providing (via a simplified model) an example of an attractive mechanism which could explain our accuracy about realist mathematics, and arguing that no comparably attractive analog to this mechanism can be used to explain our accuracy about moral realist facts ${ }^{4}$.

\footnotetext{
${ }^{4}$ One might wonder how giving any such simplified example of an explanation (which thereby gets certain aspects of known history wrong) could suffice to answer access worries. But, one can think about access worries as presenting a 'how possibly' question ('how can we have gotten significant reliability about realist mathematics?') wherein we are challenged to reconcile the apparent reality of some state of affairs with certain obstacles (consciously recognized or not) which make this state of affairs seem impossible (c.f. [5] and [33]). And one can answer the how possibly question by giving an example of an explanation for the relevant state of affairs which is compatible with all the obstacles - even if there is little reason to believe that this explanation is true.

Indeed, one can often best answer such how possibly questions by giving a simplified explanation which gets certain details of known history wrong - provided that all blocking conditions are accommodated and the core explanatory mechanism being demonstrated is sufficiently adaptable. For a (somewhat macabre) example, note that one could could answer the 'how possibly' question 'How could it be that Irish potato consumption in the 1850s rose every time the price of potatoes increased?' by providing a simplified model on which every person in Ireland in 1850 has exactly $\$ 100$ available each day to purchase food and there are only two kinds of food sold: potatoes and beef, everyone needs 2200 calories a day and prefers beef to potatoes, and every 100 calories of beef cost $\$ 10$ while, initially, every 500 calories of potatoes cost $\$ 1$. Clearly this story contains many false elements; we know that actual Victorian shoppers behavior wasn't nearly so simple. But this unrealisticness actually helps the above story answer the 'how possibly' question, by more clearly presenting a core mechanism which accommodates all relevant blocking conditions and can plausibly be complicated and adapted to fit known historical details (See [20] for more on the value and nature of such unrealistic/idealizing explanations in the sciences.).

I will describe an analogously simplified story about human access to realist mathematical facts - one which is simplified in various ways but accommodates apparent blocking conditions
} 


\section{Apparent Contrast Between Moral and Math- ematical Realism}

Now let us turn to the contrast between moral and mathematical realism. The term 'realism' has infamously been used in many different ways by many different philosophers[43]. With this in mind, I will lay out how I will use the terms moral and mathematical realism, attempting to evoke concepts typically at issue in Companions in Guilt arguments ${ }^{5}$.

\subsection{Mathematical realism}

By mathematical realism, I mean what is sometimes called truthvalue realism, i.e., the idea that there are definite right answers to certain mathematical questions, whether we can ever discover them or not, and which we are not free to stipulate $^{6}$.

For instance, I take mathematical realism to entail that every first order sentence in the language of arithmetic (e.g., the claim there are infinitely many twin primes) is either true or false, even Gödelian statements whose truth isn't provable from our current axioms. Even if we learned that for some such sentence $P$, neither $P$ nor $\neg P$ is provable from axioms we accept, we would not be free to toss a coin and extend our current number theoretic practice by adding

like the abstractness and metaphysical necessity of mathematical facts, our lack of causal contact with mathematical objects, and our knowledge of mathematical theories in advance of any any scientific applications. Of course whether such a simplified explanation succeeds in answering a 'how possibly' question depends on the specific blocking conditions one takes to be part of that question. So someone could always re-raise the access problem for truth-value realism about mathematics by citing features of our actual phenomenology/biology/history which recreate the appearance that no explanation is possible. But I'm not aware of any way that my story differs from reality would seem to create such an impression.

${ }^{5}$ That is, I will try to indicate a shared feature of the kinds of Moral Realism which are claimed to face access worries, and a kind of Mathematical Realism which philosophers moved by access worries about morals have been reasonably loath to give up.

${ }^{6}$ This powerful and popular doctrine is usually called truth-value realism about mathematics or the idea that there are 'proof transcendent' mathematical facts. This is distinct from object realism, the doctrine that mathematical objects literally exist. Both ontologically realist and anti-realist ways of developing this doctrine have been fleshed out in the literature. 
$P$ if we got heads and $\neg P$ if we got tails. Something in our current conception of the numbers (e.g., our expectation that the numbers (presuming they exist) satisfy $P A_{2}$, already suffices to ensure that only one of these options expresses a truth $)^{7}$.

In contrast, this mathematical realism does not limit which coherent putative structures are proper objects of study. Indeed, contemporary mathematical practice seems to push in the opposite direction. For example, see Kitcher on the history of the complex numbers ${ }^{8}$, Coles's autobiographical remarks about mathematicians' apparent freedom to introduce new structures ${ }^{9}$ and Lockhart's comments about mathematical creativity ${ }^{10}$.

In this paper I will consider what people who accept mathematical realism (in the sense above) can say about access worries. But perhaps I should stress that I do not purport to argue for mathematical realism here. I simply evaluate whether the popular position of accepting mathematical realism while rejecting

\footnotetext{
${ }^{7}$ Of course, mathematicians are still free to reason about non-standard models of the numbers which make different arithmetic sentences true, but it takes more to count as talking about such a non-standard model than merely believing some statement which is false in the natural numbers but true in a non-standard model. For example they can do this by introducing a new concept which they claim only satisfies $P A$ but not all of $P A_{2}$ [11].

${ }^{8}$ Kitcher writes that early advocates of the complex numbers secured their adoption by showing that the complex numbers "would submit to recognizably arithmetical treatment [and arguing] that the new operations shared with the original paradigms a susceptibility to construal in physical terms... geometrical models of complex numbers answered to this need, construing complex addition in terms of the operation of vector displacement and complex multiplication in terms of the operation of rotation." [28] And he notes that "the metamathematical views of the practices of mathematicians up to the end of the eighteenth century contained a requirement that [which was later dropped], for any kind of number, some statements about numbers of that kind must admit of concrete construal." [28]

9 "Reflecting on my experiences as a research mathematician, three things stand out. First, the frequency and intellectual ease with which I endorsed existential pure mathematical statements and referred to mathematical entities. Second, the freedom I felt I had to introduce a new mathematical theory whose variables ranged over any mathematical entities I wished, provided it served a legitimate mathematical purpose. And third, the authority I felt I had to engage in both types of activities. Most mathematicians will recognize these features of their everyday mathematical lives." [8]

10 "II]n mathematics... things are what you want them to be. You have endless choices; there is no reality to get in your way. On the other hand, once you have made your choices (for example I might choose to make my triangle symmetrical, or not) then your new creations do what they do, whether you like it or not. This is the amazing thing about making imaginary patterns: they talk back! The triangle takes up a certain amount of its box, and I don't have any control over what that amount is. There is a number out there, maybe it's two-thirds, maybe it isn't, but I don't get to say what it is. I have to find out what it is." [31]
} 
moral realism on the basis of access worries is coherent.

\subsection{Moral Realism}

In contrast, moral realism of the kind which has raised intuitive access worries seems to require something more than mere truth-value realism. Many philosophers (like moral projectivists and sentimentalists[24]) have been quite willing to judge moral claims as either true or false without (in a sense) taking them to be 'about' anything more than social norms, sentiments etc. The property that is both responsible for moral realism's access problem and the intuitive sense that it refers to what's 'really' moral is sometimes referred to as mindindependence ${ }^{11}[41]$. But spelling out just what it means to be mind-independent in this sense is notoriously fraught (e.g., facts about human psychology and beliefs would seem to be 'mind-dependent' in a way that doesn't prevent them from being objective and separate from our recognition practices). Luckily however we don't need the full power of this notion to raise strong access worries for moral realism. The following disagreement thesis is sufficient to illustrate a substantial dissimilarity with mathematical realism.

The intuition behind the disagreement thesis is that our moral knowledge is more than a mere elaboration of definitional freedom and/or exploration of proof-transcendent facts about logical coherence and consequence. A powerful moral realist intuition holds that which laws relating moral to descriptive facts express truths should reflect something deeper than mere contingencies about what kinds of things human beings tend to feel positive emotions towards, or how we have chosen to use our words. It maintains that people with different morality-like practices (people who had analogous tendencies to pursue, repent, blame etc tied to descriptive facts in a different way) would be wrong about

\footnotetext{
${ }^{11}$ Note that mind-independent is a term of art that entails properties beyond mere intersubjectivity.
} 
morality rather than right about something of equal metaphysical interest ${ }^{12}$. Accordingly, for the purposes of this paper, I will take moral realism to require accepting the following disagreement thesis (or an analogous principled formulated by replacing permissibility with the primitive of your choice):

Disagreement Thesis: Necessarily, if two thinkers (or communities) appear to be disagreeing about the permissibility of some action (where all parties agree on the usual action guiding role of permissibility ${ }^{13}$ ) there is a single proposition which they are disagreeing about $^{14} 15$

Such speakers aren't like the protagonists in Frege's example of two people arguing about whether a coin is heavy where each is talking about the coin in his own pocket.

Projectivists about morality would reject this claim. For, on the projectivist picture the English language term "permissible" (rigidly) applies to the kind of things that bear a certain relationship to our actual moral sentiments, just like

\footnotetext{
${ }^{12}$ Horgan [23] proposes a moral twin earth thought experiment in which deontological theories best fit our moral sentiments, but consequentialist theories best fit the slightly different moral sentiments of variant humans on some twin earth. This thought experiment vividly demonstrates, fans of moral realism feel strongly that people with slightly different (but logically coherent) moral sentiments would count as having a genuine moral disagreement with us, not just as talking about some notion of shmoriality of equal metaphysical interest.

${ }^{13}$ When I talk about the characteristic action guiding role of permissibility, I don't just mean making correct verbal claims about what's "permissible". Instead I mean being suitably inclined to avoid what one judges to be "not permissible", make amends for doing it, demand compensation from others only when they do something which one classifies as "not permissible", and perhaps treating the "impermissibility" of some action as a categorical reason to avoid something rather than merely a defeasable consideration against it etc. Admittedly the task of explicitly stating exactly what the characteristic action guiding role of moral terms like 'permissible' is, is a non-trivial one. Also, see Matti Eklund's Alternative Normative Concepts [10] for discussion of some major current approaches to moral facts which would reject the disagreement thesis above, and how these can seem significantly deflationary.

14 Obviously, this claim only holds generically, and is not meant to suggest that moral philosophers never have purely verbal disputes. It is enough that sufficiently clear attempts to refer to permissibility (or your favorite moral primitive) have this property.

${ }^{15}$ One could, without harm to any of the arguments I will be making below, weaken this thesis to allow that when two people 'permissibility' with its characteristic action guiding role its not the case both are latching on to a notion of equal metaphysical interests (allowing that people with sufficiently different inclinations and sentiments - paperclip maximizers and the like - might either fail to express anything with their 'permissibility' talk, or might count as latching on some other concept of (somehow) lesser metaphysical status than permissibility.
} 
'edible' refers to those actions which bear a certain relationship to what we can digest. So beings who had different moral sentiments and/or inclinations to admire praise and blame ${ }^{16}$, and correspondingly different judgments about what is "permissible", would also count as expressing truths about a different concept PERMISSIBILITY*, with equal metaphysical status to our own.

\subsection{Contrasting Relationships to Logical Coherence}

Now let's turn to the access worries faced by moral and mathematical realists, and the plausiblity of appealing to some kind of general logical accuracy to solve them ${ }^{17}$.

Moral realists face an access problem to the extent that they are unable to explain how our beliefs about morality (such as what actions are permissible) so well match the truth about morality. They must explain why we label just the right descriptive class of actions as permissible ${ }^{18}$ since, per the Disagreement Thesis, any (substantially different) alternate practice would be wrong about PERMISSIBILITY rather than right about some other concept.

At first glance, it might seem mathematical realists face the same challenge.

\footnotetext{
${ }^{16}$ Here I mean creatures who feel inclinations to resent, admire, regret, check their impulses etc. which are phenomenologically and behaviorally similar to our own, but relate to descriptive facts very differently.

${ }^{17}$ One might worry that an argument like the one Clarke-Doane provides in [26] suggesting there isn't a determinate notion of metaphysical possibility could be raised against logical possibility. In what follows I will tend to assume there is a single determinate notion of logical possibility (much as I think there is a determinate notion of physical, and perhaps metaphysical, possibility). I take defending that view to be beyond the scope of this paper, since all that defusing the companions in guilt argument requires is a plausible route to alleviating access worries for mathematical but not moral realism.

Let me also note that my story doesn't, strictly speaking, require us to say that there's a single notion of logical possibility. It just has to be that the mechanisms above can reliably give us accuracy about some notion of logical possibility which is sufficiently narrow to both rule out all the mathematical posits one takes to be bad, and imply that sufficiently many further claims about these structures (all the ones one takes to have definite truth conditions) are logically are necessary consequences. Perhaps notions of logical possibility based on various kinds of non-classical logic can also do this job, especially for readers who have more generous intuitions about what mathematical structures can succeed/less vigorously realist intuitions about what proof transcendent truths must obtain concerning a given structure.

${ }^{18}$ That is, they must explain our managing to form moral concepts which bundle together just this particular descriptive set of actions with permissibility's characteristic action guiding role.
} 
After all, they must explain why our beliefs about arithmetic sentences are true rather than false, even though there are logically coherent structures in which these sentences turn out to have a different truth values. For example, there is (on the, presumably true, assumption that PA is consistent) a model of the integers satisfying the first order axioms of arithmetic (PA) in which Con $(P A)$ turns out to be false. Thus, it might seem that the mathematical realist's position regarding our beliefs about arithmetic is very similar to the moral realist's position regarding our beliefs about permissibility.

However, unlike the moral realist, the mathematical realist is free to appeal to our choice of topic in this explanation and say that any that logically coherent ${ }^{19}$ variant on our arithmetical practice would have expressed a truth. Specifically, the mathematical realist can appeal to the fact that we take the numbers to satisfy $\mathrm{P} A_{2}$ (a categorical second order axiomatization of the natural numbers ${ }^{20}$ ) to specify the structure under investigation in a way that determines the truth or falsity of all arithmetical sentences. Importantly, the mathematical realist need not explain why we decided to investigate $P A_{2}$ rather than some other structure. They can say that any logically coherent description of structures for mathematical investigation would succeed ${ }^{21}$, but given that we accept $P A_{2}$ there are definite right answers to all questions which are logically necessitated by $P A_{2}$.

This is a critical difference. While the mathematical realist only needs to explain how we came to accept a some logically coherent characterization of

\footnotetext{
${ }^{19}$ I think requiring that successful mathematical posits be classically consistent provides the best fit with the combination of definite right answers and freedom to stipulate which are found in mainstream contemporary mathematical practice. Even among those who question classical logic, it's surprisingly widely accepted in the contemporary (as opposed to early 20th century) analytic philosophy literature that mathematics is one of the areas where classical logic is correct [15][22].

${ }^{20}$ As a categorical axiomatization, $P A_{2}$ (unlike $P A$ ) admits no non-standard models.

${ }^{21}$ Though see Appendix A for a caveat about what happens if we depart from normal (pure) mathematical practice by allowing stipulations characterizing mathematical structures to employ unrestricted quantification over the total universe (including non-mathematical objects)
} 
'the numbers' and derive our beliefs from that characterization, the moral realist must explain not only how we work out the consequences of some particular permissibility-adjacent concept but why, of all the logically coherent permissibility-like concepts ${ }^{22}$, we choose the right one.

This doesn't make the access problem trivial for the mathematical realist. That is, one might worry that our ability to postulate coherent, rather than incoherent, structures (and in some cases our ability to recognize that stipulations are categorical ${ }^{23}$ ) itself raises an access problem.

Also, as such a categorical description must necessarily be non-first-order, the mathematical realist will need to invoke a notion of logical coherence and consequence for non-first order descriptions of states of affairs. This requires that we embrace a notion of logical coherence which is distinct from the mere inability to derive a contradiction in some (computably specified) deductive system $^{24}$. But embracing such a powerful syntax transcendent notion of logic is fairly popular and independently $\operatorname{motivated}^{25}$.

\footnotetext{
${ }^{22}$ That is, of all the possible subsets of descriptive characterizations of actions, how do we choose the right subset to identify with the permissible actions (in the sense of applying them in permissibility's action guiding role). By the Disagreement Thesis the realist is committed to there being only a single such subset which, when used to guide our actions in the way we use permissibility, yields truths of equal metaphysical status.

${ }^{23}$ Note also that categoricity claims can be expressed as logical coherence claims in the powerful sense invoked here (is it logically necessary that if two objects have the relevant structure than some second order relation variable couldn't pair them up in a certain way), so even if you say that all mathematical posits have to be categorical there is no problem.

${ }^{24}$ The contrast between mere syntactic consistency (impossibility of deriving a contradiction) and the fuller notion of 'semantic' consistency being invoked here can be dramatized by thinking about semantic consistency as being witnessed by set theoretic models (it's a surprising fact that, by the completeness theorem, these two turn out to agree in the case of first order logic). Appealing to such set existence claims to define our concept of logical possibility could raise problems for the project of explaining mathematical accuracy in terms of logical accuracy considered here. But as Hartry Field as nicely emphasized in [15] [12], we seem to have a separate notion of logical coherence associated with our concept of validity, which is prior to our understanding of claims about set theoretic models and has no dependence on it.

${ }^{25}$ At first glance, one might be tempted to identify claims about logical coherence and consequence (aka validity) with claims about the existence of a set theoretic model. However, philosophers like Field have convincingly argued that, "We should think of the intuitive notion of validity not as literally defined by the model theoretic account, or in any other manner; rather, we should think of it as a primitive notion." [15]. Very crudely, the issue is this: a key aspect of our notion of logical coherence is that what's actual must be logically possible. But, if we identify logical possibility with the existence of a set theoretic model then it seems puzzling why the inference from actual to possible is justified, since the total universe can't be
} 
This brings us to the following (crude) example of the apparent contrast between the relationship of mathematics and morals' to logic:

- The mathematical realist can (and usually does) say: nearly all (conservative $^{26}$ ) logically coherent (in the sense above) variants on our number practices would express a truth of equal metaphysical status to the one we currently express - if perhaps with different pragmatic usefulness or aesthetic interest.

- The moral realist will say ${ }^{27}$ : almost no (conservative) logically coherent variants on our permissibility practices would express a truth of equal metaphysical status to the ones we currently express.

\subsection{Support from the Philosophy of Mathematics Litera- ture}

Further support for the idea that realist mathematical accuracy could be (somehow) attractively explained in terms of mere accuracy about logical coherence is provided by the existence of a number of contemporary philosophical views which converge on this point. These views (forming, what we might call, the structuralist consensus) are committed to a close relationship between logical coherence and mathematical fact. Such views endorse the above mathematical realist idea that there are definite proof transcendent right answers to mathematical questions, while taking mathematics to be 'the science of structure', and maintain that any (or nearly any) choice of new mathematical structures coherently extending one's current mathematical practice would succeed ${ }^{28}$. I have

represented as a set theoretic model (because, assuming realism about set theory, the universe contains all the sets). Field, Rayo and Hellman all invoke such a proof transcendent notion of logical coherence (or the corresponding notion of logical consequence) in [13], [35] and [21]. ${ }^{26}$ See Appendix A for much more detail on this.

${ }^{27}$ See the discussion of how this applies to Kantians below.

${ }^{28}$ Of course, it is the whole of one's practice that must be coherent. So even coherent posits might be unacceptable if they are not jointly coherent with existing posits. Acceptable 
in mind views like classic Set Theoretic Foundationalism and other truth-value realist forms of Plenetudinous Platonism, Neo-Fregeianism, Geoffery Hellman's modal structuralism[21], Stuart Shaprio's ante rem structuralism[38], Quantifier Variance fueled neo-Carnapian realism about mathematical objects, and Fictionalism $^{2930}$.

As these views allow any coherent mathematical structure ${ }^{31}$ to be posited, they transform an explanation of our access to logical coherence facts into an explanation of our access to mathematical facts. For if we take our ability to postulate logically coherent, rather than incoherent, mathematical structures

mathematical posits must also not employ unrestricted quantifiers in a way that imposes constraints on the size of the non-mathematical universe. See appendix A for more details.

${ }^{29}$ Some of these views on the semantics of mathematical claims are hermeneutic and others are revisionary, to use Burgess and Rosen's terminology [4]. (Hermeneutic views present accounts of what we actually mean, revisionary views present accounts of what we should mean/how we should revise our practices.) I won't stress the difference here, because it won't mater to the genesis or solution of the access worries discussed here.

${ }^{30}$ All the views in the structuralist consensus discussed above say that mathematical posits have to be classically consistent. Doing this lets them secure the truthvalue realist intuition noted above: that we are not free to extend $P A_{2}$ by stipulating either answer to questions which our proof practice will decide. For $P A_{2}$ is categorical, so only one option for whether to add an arithmetical sentence $\phi$ or $\neg \phi$ will be classically consistent. Embracing the requirement that acceptable mathematical structures must be classically consistent doesn't seem to prevent them from capturing all mainstream mathematics. For example there is no central aspect of mainstream mathematics which is generally taken to require positing a logically incoherent structure (though some philosophers like Graham Priest[34] have argued that set theory requires using paraconsistent logic, this is very much a minority view). However, as noted above, I don't pretend to argue for this level of truth value realism about mathematics or this classical logical consistency constraint here.

Philosophers who are inclined to say that mathematical posits only need to be 'logically coherent' with regard to some weaker logic, a simple variant on the strategy I propose for answering access worries for truth-value realists can also be used by those who favor weaker logics and less realist (in the above sense) conceptions about mathematics to answer their own access worries in an internally coherent fashion. Plausibly there is a notion of $X$-consistent logical possibility which is built up from $X$-consistent logic (where this is weaker than classical logic) and one could argue that any $X$-coherent (i.e., $X$-consistent logically possible) mathematical posit succeeds relative to the corresponding less realist conception of mathematical truth built from $X$-consistent logic. If so then one could plausibly avail oneself of a similar, if not identical (as my account of generalizing from concreta seems to build in classical logic) story explaining how we can have access to $X$-consistent logical possibility.

${ }^{31}$ Note this view is compatible with the widespread view among mathematicians that there is a nontrivial search for the correct axioms for set theory. For once we allow rich second order conceptions of mathematical structures (like $\mathrm{PA}_{2}$ or $\mathrm{ZFC}_{2}$ ), our conception of a structure can logically necessitate things which transcend what our proof procedures allow us to recognize. On this view, the search for new axioms can be understood as an attempt to find first order axioms which follow from our non-first order conception of set. For even when, as with AC and $\neg \mathrm{AC}$ (or con(PA) and $\neg \operatorname{con}(\mathrm{PA})$ ), both alternatives are known to be syntactically consistent with the standard first order theory (ZFC or PA), our full/second order conception of the relevant mathematical structure may only allow one of these to be consistently added. 
for granted, mathematical knowledge flows simply from application of logical inferences (which are themselves expressible as claims about coherence ${ }^{32}$ ).

To see how this plays out in more detail, remember that resolving Field's access problem requires explaining why 'if mathematicians believe that $\mathrm{p}$ then p' holds reliably (where p ranges over mathematical claims). Now any explanation of our access to logical coherence facts, i.e., why reliably (descriptions of) structures we believe to be coherent (incoherent) are actually coherent (incoherent), gives us an explanation of why mathematicians only study coherent posits. By definition, views in the Modal Structuralist consensus imply that one can express truths by adopting any ${ }^{33}$ coherent mathematical posit and deriving logical consequences from it ${ }^{34}$. So this suffices to explain the desired reliability claim $^{35}$.

For example, Plenitudinous Platonists and (many) Neo-Fregeans think that nearly all coherent mathematical descriptions $D$ which mathematicians are likely to consider will truly describe some portion of the mathematical universe (and that all our further purely mathematical knowledge can be gained by recognizing the logical consequences of $D$ ). Ante rem Structualists like Shapiro believe that there are a wide range of special abstract objects, called structures, corresponding to different coherent descriptions, like the second order description of the natural numbers $P A_{2}$ mentioned above ${ }^{36}$.

\footnotetext{
${ }^{32}$ The inference from $\phi$ to $\psi$ is logically valid iff it is not logically coherent that $\phi$ and $\neg \psi$.

${ }^{33}$ Technically Plenitudinous Platonists may deny that some coherent but abstruse mathematical posits would express truths but not any posits encountered in the normal course of mathematics. Also see Appendix A

${ }^{34}$ The assumption of access to logical coherence facts ensures that reliably claims mathematicians believe to be logical consequences of a posit are logical consequences of that posit.

${ }^{35}$ This explains mathematicians' accuracy and thereby solves Field's access problem even if most mathematicians don't dabble in philosophy or and are unaware of the structuralist consensus. For note that Field's access problem arises from the impression that the realist must (and cannot) explain human reliability about mathematics in an internally coherent way. Thus it can be answered by providing an attractive realism-compatible explanation for human accuracy about mathematics, regardless of whether actual mathematicians believe this theory. Of course, if mathematicians do worry about the access problem it is only by realizing that any coherent mathematical structure is a valid subject of study that they can resolve those worries.

${ }^{36}$ Shapiro appeals to a form of quantifier variance to address standard worries about the
} 
Modal Structuralists like Hellman take knowledge of pure mathematics to just be knowledge of logical coherence claims: knowledge that it is coherent for objects to satisfy some mathematical description and logically necessary ${ }^{37}$ for objects satisfying this description to also satisfy some sentence $\phi$. For example, if $P A_{2}$ is a sentence in second order logic which uniquely describes the the natural numbers and $\phi$ is a sentence in the language of arithmetic, then the modal structuralist can render the intended meaning of $\phi$ as 'it is logically possible that $P A_{2}$ and logically necessary that if $P A_{2}$ then $\phi^{\prime}$. Similarly, Fictionalists say that mathematical existence statements may all be literally false, but take the correctness conditions for asserting $\phi$ in the fiction to be essentially the same as the truth conditions given by Hellman. ${ }^{38}$

Now one might object that mathematicians don't make explicit stipulations introducing new mathematical structures, or accept any view within the Structuralist Consensus for use in reasoning about which mathematical stipulations can succeed. Thus, one can't appeal to the idea of mathematicians explicitly reasoning as above to answer access worries. However, (I claim that) if the structuralist consensus is true, then then mathematicians plausibly gain true beliefs by introducing mathematical structures in a way that is endorsed the structuralist consensus, even though they don't accept any particular theory about why introducing mathematical structures in the way that they do is acceptable.

As I highlighted in section 3.1, contemporary mathematical practice seems to allow mathematicians significant freedom to introduce new kinds of mathematical objects, such as complex numbers, sets and the objects and arrows of category theory. If this process is actually reliable (as views within the structuralist consensus can say that it is), then it seems plausible that mathemati-

consistency of positing objects corresponding to all possible coherent descriptions of mathematical structures. See [39].

${ }^{37} \mathrm{As}$ usual $\phi$ is logically necessary just if it is not logically coherent that $\neg \phi$.

${ }^{38}$ See [2] for my preferred Quantifier Variance account of how mere accuracy about logical coherence could be used to explain accuracy about mathematics. 
cians have defeasable default warrant (of the kind advocated in [14] and [1]) for using it, and they can gain knowledge by using it without having to be able to explain why it's reliable, much like with other belief forming methods such as sight or logical deduction ${ }^{39}$. (Though maybe if mathematicians do get worried they have to answer access worries, but can do so by accepting the philosophical arguments I have proposed).

\section{The Access Problem For Logical Coherence}

\subsection{The question}

So far I've tried to motivate the idea that one can plausibly explain our accuracy about mathematics in terms of accuracy about (a suitably powerful notion of) logical coherence. But does this help with access worries?

Many philosophers have felt that (some kind of) 'logical knowledge' is somehow specially unproblematic or immune to access worries. But, even if you accept this, you might not accept that the kind of knowledge needed to explain mathematical accuracy counts as logical knowledge in the relevant sense ${ }^{40}$.

\footnotetext{
${ }^{39}$ For example, consider how things look to the Quantifier Variantist who says that one can make ontologically inflationary stipulative definitions which change the meaning of our quantifiers (not just the extensions of predicates) to ensure their own truth.

It is a common idea that our acceptance of some claims (e.g., about our knowledge that bachelors are unmarried men, or of which shades of red qualify as crimson) can behave like a stipulative definition for the sake of answering access worries. Call this giving a metasemantic explanation for our knowledge of some facts about how the properties of bachelorhood or crimsoness applies. The Quantifier Variantist explanation above is directly analogous to the explanation non-Quantifier Variantists have for our accuracy about about mundane principles like 'all bachelors are unmarried'

We don't generally take the acceptability of giving such a metasemantic explanation for a person's accuracy about certain facts to require that they have a correct theory of metasemantics (e.g. either which explicit stipulations succeed, or when behavior can function like a stipulative definition). It just needs to be the case that their behavior reliably falls within the parameters that would be adopted by someone who did have a conscious theory of these things. Thus it seems plausible to say that if ontologically inflationary stipulative definitions are possible, then someone can succeed in making one and gaining knowledge from it if they can reliably succeed in making good/avoid making bad ones, even if they don't have an explicit theory about what it takes for ontologically inflationary stipulative definitions to succeed.

${ }^{40}$ Note that when considering knowledge of second order logical coherence we are forced to drop many features of knowledge of purely first order logical coherence (e.g., the existence of
} 
Thus, we can't simply rely on these intuitions to address residual access worries about the kind of logical knowledge needed to explain our mathematical $\operatorname{accuracy}^{41}$.

One strategy for addressing this worry has been to conceptually analyze the notion of $\operatorname{logic}^{42}$. Happily however, we don't need to settle this vexed question to evaluate companions in guilt arguments. For our purposes it doesn't matter whether modal logic, second order logic, or logical coherence are really logic. What's needed for our argument is just for there to be some notion of logical coherence/knowledge which does the needed work, i.e., some notion such that one can plausibly explain accuracy about mathematics largely in terms of accuracy about it and its access problem looks relatively tractable.

The rest of this section will be devoted to suggesting that access worries about logical coherence (suitably understood) are tractable. I will defend the intuition that our knowledge of facts about logical coherence (even the logical coherence of second order statements) creates less of an access problem than moral realist knowledge initially appear to, by discussing some promising mechanisms for explaining our accuracy about logical coherence.

\subsection{Some Mechanisms and a Toy Model}

To appreciate the scope of what needs to be explained, note that merely using the correct introduction and elimination rules for first order logic does not allow one to recognize the positive fact that a scenario is logically coherent. For example, first order introduction and elimination rules don't allow one to

a proof procedure which lets one recognize all logical incoherence eventually - as guaranteed by the completeness theorem - and an uncontroversially domain-neutral logical vocabulary - $\wedge, \vee, \neg, \exists, \forall$ which is used to talk about everything) which might seem relevant to easing access worries.

${ }^{41}$ See [29] for a version of this concern.

${ }^{42}$ For example, we might say that logic must be topic neutral and then note that the kind of logical knowledge invoked above qualifies, insofar as it tracks the most general patterns in how any objects can be related by any relations. 
recognize that it would be logically coherent for there to be two distinct things $(\exists x)(\exists y) \neg x=y^{43}$.

So, imagine people who begin with the ability to use standard first order language to describe their macroscopic physical environment (something which people on all sides of companions in guilt worries are willing to take for granted). Further suppose that they have some concept of scenarios being logically coherent or not, i.e., possible or impossible with regard to 'the most general constraints on how any objects can be related by any relations ${ }^{\wedge 445}$ but have little or no knowledge that anything is logically possible or impossible. How could they non-miraculously acquire powerful methods of reasoning about logical possibility sufficient to capture much of contemporary mathematics?

I want to suggest a story along the following lines. As inquirers, we attempt to predict and explain the behavior of concrete objects. There are more and less economical ways of doing so ${ }^{46}$. When we are dealing with sufficiently diverse and plentiful collections of concrete objects, the most economical explanations for regularities may well appeal to a combination of general principles which constrain how any objects can be related by any relations, and specific physical or metaphysical laws whose application is restricted to certain particular kinds of objects or relations.

\footnotetext{
${ }^{43}$ By Gödel's completeness theorem[18] it turn out that every logically incoherent first order scenario allows for a derivation of contradiction using the usual inference rules for first order logic. But this was a substantive result which it took real mathematics to prove, so not something the denizens of our story would or could assume. Furthermore, humans clearly don't infer that a scenario is logically coherent by checking all possible proofs (whose premises are true in the scenario) for a contradiction.

${ }^{44}$ I take this general way of thinking about the nature of logic from Frege[16] and the larger literature on the supposed topic neutrality of logic e.g. [40].

${ }^{45}$ In essence, the mechanisms proposed below are supposed to explain how one could go from acceptance of very limited principles to acceptance of much more powerful principles of reasoning about logical possibility (as required to make sense of modern mathematics).

${ }^{46}$ Note that our compositional language (and thoughts) allows us to formulate many syntactically different descriptions of logically impossible states of affairs. Thus, many plans which we can verbally represent can be discarded as physically impossible purely on the grounds that they require something logically impossible. Recognizing general laws of logical possibility helps systematically and efficiently avoid wasting time on plans that are logically impossible.
} 
I will suggest that pressure to efficiently predict what is practically possible in situations of evolutionary interest can help explain how creatures like us could have gotten correct methods of reasoning about logical possibility. One can think about our general methods of reasoning about logical possibility as being susceptible to improvement and correction by the world in three ways.

On the one hand, pressure to acknowledge facts about concreta encourages us to accept the logical coherence of certain things. For example, imagine that you aren't sure whether the first order state of affairs described by some mathematical hypothesis involving relations $P, Q$, and $R$ is logically possible. If I then point out that the relations of friendship, nephew-hood and having been in military service together apply in just this way to the royal family of Sweden, this will cause you to accept that the scenario in question is logically possible.

On the other hand, our need to elegantly explain regularities in the world creates pressure to conclude certain states of affairs are logically incoherent. Suppose, for example, that someone thought it was logically coherent for 9 items to differ from one another in which of three properties they had, e.g., for 9 people to choose different combinations of sundae toppings from a sundae bar containing three toppings. This person would have to explain the striking lawlike regularity that, regardless of the type of items and properties in question, we never wind up observing more than 8 such items. They might postulate new physical regularities to explain why apparently random processes of flipping three coins never generated the forbidden 9th possible outcome. However, this explanation (or some analogous one) would have to apply at every physical scale we can observe, from relationships between the tiniest particles to relationships between planets and stars (as well as to less concrete objects like poems and countries). A much more elegant explanation is that the unrealized outcome isn't logically coherent. Recognizing that the forbidden 9th outcome is forbidden 
in all possible domains is much more efficient than hypothesizing separate laws prohibiting it in each specific situation (and thus there is pressure to do so).

Accordingly, we can think of facts about what's actual as simultaneously a useful source of data about what's logically possible, physically possible, chemically possible, etc. We try to efficiently predict what will happen by patching together laws with different levels of generality. Though we face an in principle choice about whether to explain any specific regularity in terms of logical necessity vs. physical law, metaphysical necessity or mere ceterus paribus regularity, patterns in our experience can still motivate attributing a noted regularity to logical necessity rather than physical law. For, as noted in the case above, if the right explanation for some regularity is that it holds as a matter of logical necessity, we should expect to see that all substitution instances of it (i.e., all sentences with the same logical structure) are true, whereas we would expect the opposite if some principle holds as a matter of merely metaphysical necessity or physical necessity.

Finally, one should note that the pressures mentioned above don't exist in isolation. Rather, the resulting beliefs (and inference methods) will be further corrected by interaction with one another. If one accepts the above story about how we could have gotten some initial 'data points' about logical possibility from our knowledge of the concrete world, one can then appeal to familiar processes of reflecting on our beliefs and recognizing when they conflict or cohere with one another to explain further improvements in our accuracy.

Once some methods of reasoning come to strike us as initially attractive via the two mechanisms above, we can arrive at new more powerful laws (just as we do in the sciences) by considering how they unify and explain these methods of reasoning. For example, in mathematics we can reliably add new axioms by choosing principles which unify and explain the mathematical beliefs which we 
already have[30]. As Gödel puts it, "There might exist axioms so abundant in their verifiable consequences, shedding so much light upon a whole field, and yielding such powerful methods for solving problems... that, no matter whether or not they are intrinsically necessary, they would have to be accepted at least in the same sense as any well-established physical theory"[19]. If this is true, then it also seems plausible that the creatures in this story might reliably expand an initial collection of good methods of reasoning about logical coherence in the same way. Moreover, when we make incorrect generalizations these can be corrected by coming into conflict with well-entrenched and concretely motivated general principles.

Note that that the kind of elegant generalization which we see in the sciences (and which I want to invoke) goes beyond simple inferences like 'the sun rose every day for the past billion years, so it will rise tomorrow.' It can include seemingly astonishing leaps, like when astronomers go from observations of points of light in the night sky to a whole model of how the planets are arranged.

Also note that our deployment of these general principles can involve thinking about how it would be logically possible for objects satisfying one description, e.g., $P A_{2}$, to exist within a larger universe - and that such reasoning can lead one to new conclusions about the original structure. For example, general principles might cause you to believe that it is coherent for there to be a copy of the natural numbers inside of a structure satisfying $Z F C_{2}$ and, by applying the generally valid inference methods (developed as above) to this structure, thereby conclude that the natural numbers satisfy the Paris-Harrington principle[27]. Since there are models of the first order version of $P A_{2}$ in which the ParisHarrington theorem is false, such reasoning can be thought of as working out the second order consequences of $P A_{2}$.

Once we have good methods of reasoning about logical coherence we can use 
these to recognize choose logically coherent/compatible (putative) mathematical structures.

Admittedly a tiny issue remains. The examples of initial data used above all involved first order states of affairs, and thus plausibly generated good principles for reasoning about which such states of affairs are logically possible. But what about modal knowledge involving second order claims - which we need to account for knowledge of the logical coherence of second order states of affairs like $P A_{2}$ as well?

If we could presume some initial knowledge about (actual) concrete second order states of affairs, then we could feed it in to the generalization mechanism above to get good methods of reasoning about which second order states of affairs are logically possible. One might say that we get pressure to recognize that there is (something like) a second order collection $\mathrm{X}$ of some first order objects (say, the cats) satisfying $\phi$ from noticing that the white cats $\phi$, or that it is physically possible to paint some cats white so that the white cats would satisfy $\phi$. And we can get pressure to recognize that there isn't any second order collection $\mathrm{X}$ of some objects which satisfies $\psi$, by way of this being the most elegant explanation for why it is physically impossible to paint some of the cats white so that the white cats $\psi$.

Developing this idea is not trivial ${ }^{47}$. However, since my goal here is just to argue that (contra companions in guilt arguments) one can rationally think the 'structuralist' program of reducing mathematical access worries to access worries about logical possibility and then solving the latter is more promising than Kantianism rather than to complete this program, I won't say more about these issues involving second order claims here.

\footnotetext{
${ }^{47}$ For example, long-standing controversies over the ontological commitments of second order quantification raise concerns over the use of second order logic in this program. I propose a solution this problem in [3] by using a more purely and intuitively modal concept to do the same work as second order logic does in articulating categorical conceptions of mathematical structures.
} 


\section{Objections}

\subsection{Logical Coherence and Large Collections}

I will conclude by considering some objections.

First, one might worry that the above story can't account for our apparent knowledge of facts about logical coherence (and necessity) involving large infinite collections.

An objector might allow that one can explain our accuracy in reasoning about countably infinite collections as above. But capturing intuitively correct truth conditions for statements of set theory (via the structuralist consensus) requires evaluating claims about the logical coherence of scenarios involving uncountably many objects. Thus, one might worry that principles of reasoning which are shaped to elegantly predict and explain what is logically coherent for finite and countably infinite collections cannot account for the degree of logical (and hence mathematical) knowledge which we actually have.

A critic might advance the following analogy: saying that elegant generalization from facts about finite and countable collections (such as physical objects and segments of space) yields principles which accurately describe what is logically coherent for some of the larger collections considered in pure mathematics is like saying that inference to the best explanation plus observations of birds in New Mexico explains our possession of true beliefs about birds in Canada as well. Presumably, in the ornithological case, we need to go gather more data in order to get many true beliefs about birds in Canada. But, in the mathematical case, we can't gather more data. Thus, our apparent possession of substantial true beliefs about what is logically coherent for larger infinite collections remains mysterious on the story I have sketched above.

I want to respond to this worry by accepting the analogy about birds above and saying that it fits the current state of human knowledge with regard to 
facts about the higher infinite rather well. Even in the case of birds, we can arrive at some true beliefs about birds in Canada just by inference to the best explanation from the facts about the birds in New Mexico. If we discovered tomorrow that some new island which had never yet been visited by explorers contained birds, I think we would reasonably expect many facts to carry over: any birds on that island would breathe oxygen, that they would have hollow bones etc. Our expectations about birds on this island would just be more sparse and less confident than our beliefs about birds in locations that we have observed.

But, this is just what happens with our beliefs about logical coherence and large collections: as one moves from logical coherence facts concerning finite collections to those concerning countably infinite collections (like the natural numbers), and then uncountable collections (like the sets) our beliefs do get more sparse and less confident. For example, the continuum hypothesis ${ }^{48}(\mathrm{CH})$ is a fairly simple statement involving sets of (relatively) small infinite size, yet it is known that both the truth and the falsity of $\mathrm{CH}$ are compatible with ZFC. Our beliefs about what large infinite collections of objects and relations are logically coherent are also frequently less confident than our beliefs about what finite and countable collections of objects are logically coherent. Sociologically, mathematicians are frequently much more confident in their claims about numbers, sets of numbers and sets of sets of numbers than in the distinctive claims of set theory about what much larger patterns of mathematical objects would have to be like ${ }^{49}$.

Thus, I think this last worry points to something that is an attractive feature rather than a flaw of the account at hand: it explains why we have relatively

\footnotetext{
${ }^{48}$ The continuum hypothesis states that there are no sets whose cardinality is intermediate between the cardinality of the real numbers and that of the natural numbers. See [25] pg 176-186 for the proof that the continuum hypothesis is independent of the Zermelo-Fraenkel axioms.

${ }^{49}$ Think of choice vs. determinacy and disputes over large cardinal axioms.
} 
sparse beliefs about what's logically possible with respect to large collections, and hence relatively sparse beliefs about the corresponding facts concerning higher set theory.

\subsection{Contrast With Quine}

There is obviously a certain similarity between my proposal and Quine's famous empiricism about mathematics (if the latter is read as an answer to access worries as well as a claim about what justifies mathematical beliefs). For example, we both invoke dealings with concreta as part of a (potential) explanation for human accuracy about mathematics. So one might wonder whether existing (fairly persuasive) objections to Quine's proposal also apply to my proposal. I will now attempt to answer this question by reviewing some important ways in which my proposal differs from Quine's and how those differences allow me to avoid some of the most troubling objections to Quine's approach ${ }^{50}$.

First, where Quine's proposal takes dealings with concrete objects to push us to recognize the existence of the particular mathematical structures which we use in the sciences, my story takes dealings with concrete objects to push us to accept correct general inference methods which can be used to derive the logical possibility of the structures we use in mathematics. Because my story makes the relationship between scientific and mathematical beliefs indirect in this way, it naturally avoids the 'problem of recreational mathematics' that besets Quine, i.e., the fact that we seem to know things about mathematical objects which are scientifically useless (like sets in the higher reaches of set theory).

My story also allows for the fact that (as emphasized by Michael Friedman[17])

\footnotetext{
${ }^{50}$ I claim that either Quine's proposal fails to solve the access problem (as the historical features it fails to account for always counted as blocking conditions in the sense of footnote 3 ) or it succeeds in meeting some initial access problem only to fall victim to a secondary access problem about how we can have knowledge given these features. In contrast, I hope the reader will share my intuition that nothing prevents sufficient scientific/historical diligence from yielding some variant on my approach from accommodating any actual facts which might appear to be blocking conditions.
} 
even in cases where mathematical structures do get quantified over in physical theories, mathematicians appear to acquire significant knowledge of these mathematical objects before a use is found for them in physics. And it makes good sense of the apparent cavalierness of both physicists and mathematicians with regard to positing new mathematical structures ${ }^{51}$. For one can say that (in such cases) mathematicians and physicists are usually already convinced of general methods of reasoning which let them derive the logical possibility of suitable structures (due to prior experiences and perhaps selection on an evolutionary time scale), and, if one of the views in the structuralist consensus is true, this is enough for them to correctly ${ }^{52}$ use such a structure.

Second, where Quine's story appeals to continuing indispensability mine appeals to past usefulness. If (as Field argues in the case of Newtonian Mechanics[12]) all quantification over mathematical structures in physics is ultimately dispensable, this would be a problem for Quine's empiricism but not for my proposal. All that is necessary for my story to work is that recognizing the logical possibility or impossibility of various claimed patterns of relationships between concrete objects was practically useful at whatever time our dispositions to reason about logical possibility were formed. Third, while Quine says mathematical knowledge is empirical, my explanatory story is entirely compatible with mathematical knowledge being a priori ${ }^{53}$.

\footnotetext{
${ }^{51}$ As Justin Clark-Doane notes, physicists appear to make new mathematical postulates much more freely than they make new physical postulates - which seems odd on a Quinean picture where our acceptance of both types of objects is motivated by the same kind of inference to the best explanation. Mathematicians seem equally cavalier about positing new objects in cases where there is reason to think the relevant structure is logically possible [6].

${ }^{52}$ More technically (remembering the case of fictionalism) this is enough for them to be as correct as any use of mathematics is taken to be.

${ }^{53}$ Consider a classic foundationalist understanding of the a priori, which traces all a priori knowledge back to some basic principles and inferences which we can be warranted in making without justificatory appeal to anything else. If you think that any beliefs can qualify as basic a priori knowledge, beliefs directly produced by the application of correct general methods of reasoning about logical possibility which we find immediately compelling (and are perhaps even innately hardwired to find unquestionable) seem like an obvious candidate.
} 


\subsection{The Kantian Program}

Finally, one might accept that the answer to access worries about mathematics sketched above looks promising, but maintain that an analogous approach to moral knowledge looks equally promising.

I've suggested that if we employ a rich notion of logical coherence (treating second order quantifiers as logical vocabulary and letting coherence require more than mere syntactic consistency) then it is plausible that mathematical accuracy can be explained in terms of logical accuracy - in a way that helps with access worries.

But an objector might point out that an exactly analogous proposal for morals has been tried by Kant and his followers - and argue that this proposal looks equally promising. One can think about Kant's appeal to the categorical imperative as a way to explain our capacity for moral accuracy by appeal to something like logical coherence (practical rationality/coherence in ones maxims). And one can see Kant-influenced contemporary philosophy of action's many attempts to move beyond the desire satisfaction model of rationality as endeavoring to enrich and develop the relevant notion of logical/practical coherence in a way that would let this explanatory strategy succeed ${ }^{54}$.

Admittedly, the kind of practical coherence the Kantian takes to be sufficient for moral accuracy is not logical coherence (and certainly not the kind of logical coherence discussed in section 4.2). But the defender of moral realism might try to replace logical coherence with something else like Scanlonian reasons for action and tell a similar story. However, for that approach to succeed, they must offer a promising account of how pressure to get practical reasoning right could give us something like a general ability to detect Scanlonian reasons to the extent necessary to explain accuracy about morals.

\footnotetext{
${ }^{54}$ For, on the the desire satisfaction model of rationality even very vicious basic desires are possible and ideal coherence doesn't seem to require anything like moral goodness
} 
I admit that if this loosely Kantian approach of reducing moral knowledge to something like logical knowledge ${ }^{55}$ and the structuralist program of so reducing mathematical knowledge looked equally promising, the companions in guilt argument would be defensible. However, I don't see any powerful argument that these two philosophical research programs are equally promising.

First, Kant's idea that morality can be reduced to a kind of logical coherence is (already) wildly controversial, much more so than the idea that something like one of the structuralist consensus approaches discussed above could work. The plain fact is that, as discussed in section 3.3 above, it sure looks like an artificial intelligence bent on maximizing the number of paper clips in the universe or Hume's sensible knave could be logically coherent but wrong about realist morals. And the history of Kantian research since 1781 hasn't done much to shift this impression.

Second, note that even if we accepted such a reduction, not every way of explaining morality in terms of something like logical accuracy would be helpful in addressing access worries. Even if this first part of the Kantian program succeeds, you can't just expand your notion of logic arbitrarily without losing the intuition that access worries about that notion of logic are tractable. For example, consider a Scanlonian picture where reasons to do things (including believe things, treat people in certain ways, reject certain kinds of treatment etc.) are treated as fundamental[ $[37]^{56}$. Suppose that you say that a general faculty for 'detecting reasons' plays a similar role in explaining our moral knowledge to the one which a faculty for reasoning about logical coherence plays in explaining our

\footnotetext{
${ }^{55}$ By this I mean something like logical knowledge, in that its access problem appears solvable.

${ }^{56}$ Insofar as Scanlon rejects my disagreement thesis (as his response to Enoch in [37] suggests) he can say something analogous to what I say about mathematics about morality, but does not qualify as a moral realist in the sense relevant to this paper. However, if he accepts the disagreement thesis (as he seems more inclined to do in a forthcoming response to commentators on the book), he wouldn't be able to characterize any obvious sense in which all variant coherent moral practices would be correct, and would face a serious prima facie access problem (as he seems to acknowledge himself).
} 
mathematical knowledge. Even if some version of Scanlon's idea can succeed in explaining accuracy about morals by appeal to such a faculty this would not suffice to banish access worries.

For a question remains about how we could have gotten a faculty which detects the (objective, realist, disagreement-criterion satisfying) reasons for rejecting certain kinds of treatment and policies which Scanlon posits. There might be pressure on someone to evaluate reasons to believe in a way that tends to produce true descriptive beliefs in the evolutionary (or meme-evolutionary) environment. And there might be pressure towards accurate means-ends reasoning, 'If there is reason for me to $\phi$ and the only way for me to $\phi$ is to $\psi$ then I should $\psi$ '.

But why should selection for good faculties (in the sense above) also give us faculties that correctly detect Scanlon's supposed reasons for treating people a certain way? This seems prima facie far more mysterious than the idea that in getting that rules that elegant predict what statements are logically possible for small collections should get rules which also tend to yield true verdicts - insofar as they say anything - about large collections as well. It is far from clear that in getting a reasons-detecting faculty which is good for means-ends rationality we would also be likely to get one that tracks reference magnetic facts about permissibility $^{57}$.

\section{Conclusion}

In this paper I have attempted to defend the position that access worries for mathematics are less severe than access worries for morals - at least relative to the current state of philosophical play. Specifically, I have argued that certain

\footnotetext{
${ }^{57}$ On the other hand, I have suggested it's more plausible that, in learning to anticipate facts about the logical coherence of physically possible and useful concrete structures, we should get principles of reasoning about logical coherence which let us detect the logical coherence of larger and more complex mathematical structures as well.
} 
core intuitions necessary for something to count as moral/mathematical realism make it far easier to say that nearly all alternative 'logically coherent' mathematical practices would have been right about something else, than it would be to take an analogous position on alternative logically coherent moral practices.

Thus, there is an attractive strategy for answering access worries about mathematical realism which seems to have no (comparably plausible) analog for defending moral realism. Admittedly, this point alone does not suffice to show that the access problem for mathematics is worse than that for morals: maybe current appearances regarding the prospects for Kantianism in morals and/or the 'structuralist consensus' regarding mathematics are wrong. Or maybe there is some other, even better, strategy for addressing access worries about morals (unrelated to exploiting a connection to logic) which has no analog for mathematics.

But it does defend the current position of many philosophers, who accept mathematical realism while rejecting moral realism on the basis of access worries. Contrary to the companions in guilt argument, no hypocrisy is needed to take different positions on moral and mathematical realism on the basis of access worries. One only needs to combine a fairly common assessment of the of the relative health of two different philosophical research programs (Kantianism about ethics and the structuralist consensus about mathematics) with pessimism about finding some radically different solution to access worries for realists about morality. 


\section{A The Problem of Logically Coherent vs. 'Con- servative' Stipulations}

A well known [13][42][9] issue for all the ways of reducing access worries about mathematics to access worries about logic discussed above, is that attempts to introduce new abstracta via principles that are (separately) coherent can nonetheless conflict with one another, or imply constraints on the behavior of objects that aren't mentioned in these principles.

For example, posits saying (in the usual Fregean way) that there are exactly four things and exactly five things in the universe are both logically coherent, but not compatible with one another. Also, if either of them were taken to be true it would impose constraints on the size of the non-mathematical universe in a way that we expect mathematics not to do.

Now, this problem is not so big as it initially seems. For we can easily reconcile these facts with the idea that knowledge of logical coherence is all we need to get pure mathematics right, by saying that what needs to be coherent are total bundles of mathematical stipulations (expressing the various mathematical structures we are talking in terms of) and saying that stipulations characterizing pure mathematical structures must have their quantifiers restricted to some collection of mathematical objects (so that they cannot imply any logically contingent constraints on the non-mathematical objects).

For, it is already mathematical common practice to describe structures like the natural and real numbers via principles whose quantifiers (are implicitly) restricted to the mathematical structure being defined by these principles (or to this structure plus some old mathematical structure which it is supposed to be extending). For example Peano Arithmetic is often formulated to include the claim that everything has a successor, by people who would actively deny 
that (say) the color blue or the Great Barrier Reef has a successor. When we restrict our attention to logically possible mathematical postulates which (like $\left.P A_{2}\right)$ are quantifier restricted to the structure you are stipulatively introducing, mere logical coherence does ensure acceptability in this sense. For it will always be logically possible to supplement whatever objects you are currently talking in terms of with a model of this logically coherent system.

Admittedly, when philosophically investigating applied mathematics, we often want to appeal to impure mathematical structures (like sets with ur-elements or functions from physical object to numbers) so we will need to allow characterizations of mathematical structures that quantify over (at least some) nonmathematical objects. Some options for a notion of conservativity (which prevents impure mathematical objects from inappropriately constraining the possibilities for the non-mathematical world) have been considered in the recent-ish literature - such as Field[13]'s story about which mathematical fictions are acceptable, and Rayo[36]'s development of this story in an ontologically realist but meta-ontologically deflationist spirit ${ }^{58}$. Adopting any of these proposals lets us retain the needed claim that the knowledge needed to make acceptable mathematical postulates is still basically logical knowledge. Accordingly, talk of 'logically coherent extensions' of our mathematical practice will refer to ones that are either quantifier restricted to mathematical objects, or at least satisfy (the right way of filling in) the conservativity requirement above.

\section{References}

[1] Sharon Berry. Default reasonableness and the mathoids. Synthese, 190(17):3695-3713, 2013.

\footnotetext{
${ }^{58}$ I actually think there are some problems with Rayo's attempt to combine Field's conservativity requirement with deflationary realism about mathematical objects[35].
} 
[2] Sharon Berry. Quantifier variance and mathematicians freedom to stipulate. In Alessandro Torza, editor, Quantifiers, Quantifiers, and Quantifiers. Themes in Logic, Metaphysics and Language. (Synthese Library vol 373), pages 367-387. Springer, 2015.

[3] Sharon Berry. Default The Access Problem for Knowledge of Logical Possibility. Forthcoming, http://www.seberry.org/aplogicalposs.pdf.

[4] John P. Burgess and Gideon Rosen. A Subject with no Object. Oxford University Press, 1997.

[5] Q. Cassam. The Possibility of Knowledge. Oxford scholarship online. Clarendon Press, 2007.

[6] Justin Clarke-Doane. Moral epistemology: The mathematics analogy. Nous, 2012.

[7] Justin Clarke-Doane. Objectivity in ethics and mathematics. Proceedings of the Aristotelian Society, 3, 2015.

[8] Julien Cole. Towards an institutional account of the objectivity, necessity, and atemporality of mathematics. Philosophia Mathematica, 2013.

[9] Matti Eklund. Carnap and Ontological Pluralism, pages 130-56. Oxford University Press, 2009.

[10] Matti Eklund. Alternative normative concepts. Analytic Philosophy, 53(2):139-157, 2012.

[11] Herbert B. Enderton. Second-order and higher-order logic. In Edward N. Zalta, editor, The Stanford Encyclopedia of Philosophy. Metaphysics Research Lab, Stanford University, fall 2015 edition, 2015. 
[12] Hartry Field. Science Without Numbers: A Defense of Nominalism. Princeton University Press, 1980.

[13] Hartry Field. Realism, Mathematics \& Modality. Blackwell, 1989.

[14] Hartry Field. Recent debates about the a priori. In Tamar S. Gendler and John Hawthorne, editors, Oxford Studies in Epistemology. Oxford University Press, 2005.

[15] Hartry H. Field. Saving Truth from Paradox. Oxford University Press, March 2008.

[16] Gottleib Frege. The Foundations of Arithmetic: A Logico-Mathematical Enquiry into the Concept of Number. Northwestern University Press, 1980.

[17] Michael Friedman. Dynamics of Reason (Center for the Study of Language and Information - Lecture Notes). Center for the Study of Language and Information, 2001.

[18] Kurt Gödel. Die vollständigkeit der axiome des logischen funktionenkalküls. Monatshefte für Mathematik und Physik, 37(1):349-360, 1930.

[19] Kurt Gödel. What is cantor's continuum problem? In Kurt Gödel: Collected Works Vol. Ii, pages 176-187. Oxford University Press, 1947.

[20] Peter Godfrey-Smith. Models and fictions in science. Philosophical Studies, 143(1):101-116, 2009.

[21] Geoffrey Hellman. Mathematics Without Numbers. Oxford University Press, USA, 1994.

[22] Ole T. Hjortland. Anti-exceptionalism about logic. Philosophical Studies, 174(3):631-658, 2017. 
[23] Terence Horgan and Mark Timmons. New wave moral realism meets moral twin earth. Journal of Philosophical Research, 16:447-465, 1991.

[24] David Hume. A Treatise of Human Nature. Oxford University Press, 2000 [1739].

[25] Thomas Jech. Set Theory. Academic Press, 1978.

[26] Clarke-Doane Justin. Modal objectivity. Noûs, forthcoming.

[27] Richard Kaye. Models of Peano Arithmetic. Clarendon Press, 1991.

[28] Philip Kitcher. The Nature of Mathematical Knowledge. Oxford University Press, 1985.

[29] Peter Koellner. Truth in mathematics: The question of pluralism. In New waves in philosophy of mathematics, pages 80-116. Springer, 2009.

[30] Peter Koellner. On the question of absolute undecidability. In Kurt Gödel: Essays for His Centennial, volume 14, pages 153-188. Association for Symbolic Logic, 2010.

[31] Paul Lockhart. A mathematician's lament. Bellevue literary press New York, 2009.

[32] J. L. Mackie. Ethics: Inventing Right and Wrong. Penguin Books, 1977.

[33] Robert Nozick. Philosophical Explanations. Harvard University Press, 1981.

[34] Graham Priest, Koji Tanaka, and Zach Weber. Paraconsistent logic. In Edward N. Zalta, editor, The Stanford Encyclopedia of Philosophy. Metaphysics Research Lab, Stanford University, winter 2016 edition, 2016.

[35] Agustín Rayo. The Construction of Logical Space. Oxford University Press Uk, 2015. 
[36] Augustin Rayo. The Construction of Logical Space. Oxford University Press, 2013.

[37] T. M. Scanlon. Being Realistic About Reasons. Oxford University Press, 2014.

[38] Stuart Shapiro. Philosophy of Mathematics: Structure and Ontology. Oxford University Press, USA, 1997.

[39] Stuart Shapiro. Epistemology of mathematics: What are the questions? what count as answers? Philosophical Quarterly, 61(242):130-150, 2011.

[40] Gila Sher. Is logic in the mind or in the world? Synthese, 181(2):353-365, 2011.

[41] Sharon Street. A darwinian dilemma for realist theories of value. Philosophical Studies, 127(1):109-166, 2006.

[42] Gabriel Uzquiano. The price of universality. Phil Studies, 1996.

[43] Crispin Wright. Realism, Meaning, and Truth. Blackwell, 1993.

[44] Richard Zach. Hilbert's program. In Edward N. Zalta, editor, The Stanford Encyclopedia of Philosophy. Metaphysics Research Lab, Stanford University, spring 2016 edition, 2016. 